\section{Violência na gestação entre \\ usuárias de serviços públicos de \\ saúde da Grande São Paulo: \\ prevalência e fatores associados}

\section{Violence during pregnancy among \\ public health care users in the \\ Greater São Paulo area: prevalence and associated factors}

\section{Resumo}

O objetivo desta investigação é estimar a prevalência da violência por parceiro íntimo na gestação entre usuárias de serviços públicos de saúde da Grande São Paulo e verificar sua associação com fatores sociodemográficos, de saúde reprodutiva, sexual e mental. A estratégia metodológica deste estudo consistiu na realização de entrevistas estruturadas (questionário) com 1.922 usuárias, entre 15 e 49 anos, em 14 serviços públicos de saúde. A análise dos dados revelou que $20 \%$ das usuárias que já engravidaram $\left(\mathrm{IC}_{95 \%} 18,2\right.$ a 21,8$)$ referem algum episódio de violência por parceiro íntimo na gestação. Em análise multivariada, observou-se que 'ter sofrido violência psicológica e física perpetrada por familiar', 'início da vida sexual antes dos 19 anos', 'recusa de uso de camisinha pelo parceiro', 'Transtorno Mental Comum' e 'não coabitar com parceiro' são fatores associados à violência na gestação. Conclui-se que a alta prevalência da violência por parceiro íntimo na gestação, sua associação com diversos fatores de saúde sexual, reprodutiva e mental são resultados que indicam a relevância de se tomar a violência como questão de saúde.

Palavras-chave: Violência doméstica. Violência por parceiro íntimo. Gestação. Fatores associados.

\section{Julia Garcia Durand \\ Lilia Blima Schraiber \\ Departamento de Medicina Preventiva, Faculdade de Medicina da USP}

Agradecimentos: À FAPESP pelo apoio financeiro. À Ana Flávia D'Oliveira e Andréia Nascimento pela assessoria na análise epidemiológica.

Fonte de financiamento: Fapesp - linha políticas públicas. Processo 98/14070-9; CNPq financiamento de bolsistas

Correspondência: Lilia Blima Schraiber. Av Dr Arnaldo, n 455; $2^{\circ}$ andar, sala 2245, Cerqueira César, São Paulo, SP

CEP 01246-903. E-mail: liliabli@usp.br 


\section{Abstract}

Objectives: To examine the prevalence of IPV (intimate partner violence) during pregnancy and its association with demographic and reproductive, sexual and mental health factors among public health care users in São Paulo, Brazil. Methods: a) interview with 1,922 health care users, ages 15 to 49 , in 14 public healthcare services. Results: $20 \%\left(\mathrm{CI}_{95 \%} 18.2\right.$ to 21.8$)$ reported IPV during pregnancy. Multiple logistic regression indicated that frequent family violence, having more than 2 pregnancies, beginning sexual life before 19 years of age, partner's refusal to wear condom, mental health problems, and being single are factors associated with IPV during pregnancy. Conclusion: The high prevalence rate for IPV during pregnancy indicates that this issue should be regarded as a major public health problem.

Keywords: Domestic violence. Intimate partner violence. Pregnancy. Associated factors.

\section{Introdução}

A violência por parceiro íntimo (VPI) no período da gestação tem sido considerada importante questão de saúde por seu impacto direto e indireto na mortalidade e morbidade materno-infantil.

Apesar de amplo espectro de prevalências encontradas no mundo, muitos estudos têm mostrado que o fenômeno é freqüente e responde por questões de saúde reprodutiva de difícil tratamento. Revisões realizadas em países em desenvolvimento $^{1,2}$ mostram uma variação em relação à violência física entre 3,8 a 31,7\%, enquanto nos Estados Unidos e Canadá encontra-se uma variação de $0,9 \%$ a $20,1 \%$, estando a maior parte dela entre o espectro de $3.9 \%$ a $8.3 \%^{3-5}$. No Brasil, pesquisas com puérperas em maternidades públicas apontam taxas de VPI na gestação que variam de $7,4 \%^{6}$ e $18,2 \%^{7}$ na forma física, e $61,7 \%^{7}$ na psicológica.

A depressão durante a gestação e pósparto $^{8,9}$, abuso de substância e tabagismo, anemia, sangramento no primeiro e no segundo trimestre da gestação e baixo ganho de peso $0^{4,10,11}$ são algumas das principais complicações à saúde materna relacionadas ao fenômeno. Além disso, observa-se que as mulheres nesta situação buscam tardiamente atendimento pré-natal, em geral no terceiro trimestre da gestação $0^{2,7,12}$.

Dentre os agravos à saúde fetal associados à violência, o baixo peso ao nascer (BPN) desperta grande interesse por representar, nos países desenvolvidos, a principal causa de morte neonatal e contribuir significantemente para o desenvolvimento de morbidades na infância. Embora os estudos sejam controversos a respeito da associação entre estes dois eventos, em uma meta-análise realizada com oito estudos publicados esta hipótese foi confirmada ${ }^{11}$. Além deste agravo à saúde, a violência vem sendo relacionada à prematuridade ${ }^{11}$, ao alto número de abortos (miscarriage e abortion) ${ }^{13} \mathrm{e}$ à morte perinatal ${ }^{6,14}$.

Aspecto que reforça a gravidade deste fenômeno é a associação da violência na 
gestação ao maior risco de sofrer homicídios $^{15}$. Atualmente, em diversas cidades americanas, o homicídio representa a principal causa de morte pré e pós-natal ${ }^{1}$. McFarlane et al $^{15}$ identificam que o risco de ser vítima de tentativa de homicídio ou de homicídio completo é três vezes maior entre as mulheres que sofreram VPI na gestação do que entre aquelas que sofreram violência fora deste período, e concluem que este fenômeno deve ser visto como sinal bastante grave de violência. Estudo brasileiro sobre o tema ${ }^{16}$ corrobora esta idéia, mostrando que as mulheres que sofrem VPI na gestação têm prevalência aproximadamente duas vezes maior de VPI física grave na vida, em relação àquelas que sofrem fora da gestação.

Estes achados demonstram a necessidade de se projetar olhar sobre esta questão, que atualmente carece de atenção tanto em âmbito de pesquisa como de intervenção. Na região da Grande São Paulo, por exemplo, não foram encontrados estudos sobre este tema com populações de serviços públicos de saúde ${ }^{16}$.

Assim, no presente trabalho analisamse os principais resultados de um estudo epidemiológico de corte transversal acerca da prevalência e dos fatores associados à VPI no período da gestação, entre usuárias de serviços públicos de saúde da Grande São Paulo, na faixa etária de 15 a 49 anos. Os objetivos específicos deste trabalho são:

- Estimar a prevalência de qualquer forma de VPI durante alguma gestação;

- Identificar fatores sócio-demográficos, de saúde reprodutiva, sexual e mental associados à violência na gestação;

- Verificar se o tipo, a recorrência e a gravidade da violência perpetrada por familiar na vida estão associados à VPI na gestação.

A opção de se investigar a violência entre usuárias de serviços de saúde justifica-se pelo interesse em subsidiar informações para o desenvolvimento de estratégias de assistência a mulheres em situação de violência, em especial no âmbito pri- mário da atenção à saúde. Acredita-se que este trabalho poderá fornecer dados e promover reflexões que auxiliem profissionais e gestores de saúde a compreender estes aspectos da população que assistem.

\section{Método}

Os dados utilizados fazem parte de banco produzido em uma pesquisa realizada entre março a dezembro de 2002, que estimou a prevalência de violência contra a mulher entre usuárias de serviços de saúde da rede pública em São Paulo, com desenvolvimento de tecnologia de atendimento para o programa de saúde da mu$\operatorname{lher}^{17}$. As prevalências encontradas, excluindo-se seu estudo na gestação, para as 3.193 mulheres pesquisadas, de 15 a 49 anos, e usuárias de 19 serviços da Grande São Paulo, assim como o detalhamento do desenho do estudo e da metodologia, estão em outra publicação ${ }^{18}$. No presente trabalho, recorre-se a partes desta pesquisa relativas aos dados de 14 dos 19 serviços. A exclusão de 5 serviços relacionou-se ao objetivo de homogeneizar a amostra de usuárias quanto à forma de acesso e ao tipo de demanda. Deste modo, trabalhou-se exclusivamente com os dados referentes aos serviços do tipo ambulatorial, porta de entrada única para atendimento a mulheres no sistema público de saúde e de demanda espontânea, isto é, unidades básicas de saúde ou centros de atenção primária $^{17}$. Todos os serviços foram eleitos por conveniência e agrupados em 9 conglomerados. O cálculo do tamanho da amostra foi baseado em uma prevalência esperada de violência física por parceiro íntimo contra a mulher de $32,7 \%$, encontrada em estudo americano ${ }^{19}$, chegando-se a uma amostra de 335 usuárias para uma precisão de 0,05, por conglomerado. As usuárias foram captadas de forma sistemática à chegada ao serviço, garantindo-se por repartição proporcional ao volume de atendimento, a presença de uma semana usual de funcionamento do serviço, bem como os períodos da manhã e da tarde. 
Do total de 2.339 entrevistadas dos 14 serviços aqui considerados, foram excluídas aquelas que nunca engravidaram (409 mulheres) e aquelas em relação às quais o questionário apresentava erro de aplicação nas respostas sobre VPI na gestação (8 mulheres), chegando-se a uma amostra final de 1.922 mulheres. As mulheres elegíveis foram aquelas que tinham entre $15 \mathrm{e}$ 49 anos e se dirigiam aos serviços à procura de atendimento para si mesmas, nas áreas de saúde da mulher ou saúde do adulto.

Em todos os serviços, a questão da privacidade para a realização das entrevistas foi uma preocupação constante. Como procedimento ético, as entrevistas eram precedidas de leitura e assinatura de Consentimento Informado. Além disso, foi oferecido suporte assistencial às mulheres entrevistadas que relataram situações de violência, por meio de entrega e orientação de uso do miniguia de serviços que atendem mulheres em situação de violência. O projeto foi submetido e aprovado pela Comissão de Ética da Pesquisa da Faculdade de Medicina da USP e Hospital das Clínicas (CAPPesq) em 12/05/2000.

No presente estudo, foram utilizados dados referentes às características sociodemográficas da mulher (escolaridade, cor da pele, estrato socioeconômico ${ }^{20}$, prática religiosa, idade); a aspectos da saúde reprodutiva e sexual (idade de início das relações sexuais, número de gestações, número de filhos nascidos vivos, número de abortamentos, número de filhos nascidos mortos, número de filhos com baixo peso ao nascer - peso $<2,5 \mathrm{~kg}$ - e recusa de camisinha pelo parceiro para prevenção de Doenças Sexualmente Transmissíveis [DST]) e de saúde mental (a presença ou ausência de transtornos mentais comuns (TMC), tendo como corte para se considerar TMC oito ou mais respostas sim no SRQ-20 ${ }^{21}$ ). Também foram estudados dados acerca da freqüência, tipo e gravidade da violência cometida por familiar e por parceiro íntimo atual ou ex-parceiro. Em relação à ocorrência de abortamentos, o questionário não discriminou aqueles que ocorreram de modo espontâneo daqueles intencionalmente provocados.

A violência familiar foi estudada separadamente por tipo de violência (psicológica, física ou sexual), tipo de agressor (mãe, pai/pai adotivo, irmão e outros familiares) e recorrência dos episódios (uma, poucas ou muitas vezes na vida).

Definiu-se como parceiro íntimo qualquer marido/companheiro ou namorado que a usuária teve ao longo da vida com relação sexual. A violência por parceiro íntimo foi investigada por tipo (psicológica, física e sexual), recorrência (uma, poucas e muitas vezes) e gravidade (moderada e grave).

Para as questões relacionadas à violência por parceiro íntimo, utilizou-se um instrumento elaborado para um estudo multipaíses coordenado pela Organização Mundial da Saúde e do qual participou o Brasil. Este instrumento abordou especificamente a violência de gênero e foi elaborado em conjunto com os pesquisadores brasileiros integrantes do estudo, entre eles os autores do presente artigo ${ }^{22,23}$. Foi considerada VPI psicológica ao menos uma reposta sim às questões: seu atual marido, ou qualquer outro companheiro ou namorado (a) alguma vez, tratou você da seguinte forma: a) Insultou-a ou fez com que você se sentisse mal a respeito de si mesma? b) depreciou ou humilhou você diante de outras pessoas? c) Fez coisas para assustá-la ou intimidá-la de propósito (por exemplo, a forma como ele a olha, como ele grita, como ele quebra as coisas/objetos pessoais)? d) Ameaçou machucá-la ou alguém ou algo de que(m) você gosta?

A VPI física foi definida por qualquer resposta sim às perguntas: seu atual/marido, ou qualquer outro companheiro ou namorado (a) alguma vez, tratou você da seguinte forma: a) deu-lhe um tapa ou jogou algo em você que poderia machucála? b) Empurrou-a ou deu-lhe um tranco/ chacoalhão? c) Machucou-a com um soco ou com algum objeto? d) Deu-lhe um chute, arrastou ou surrou você? e) Estrangu- 
lou ou queimou você de propósito? f) Ameaçou usar ou realmente usou arma de fogo, faca ou outro tipo de arma contra você?

A VPI sexual foi considerada alguma resposta sim às perguntas: seu atual marido, ou qualquer outro companheiro ou namorado(a) alguma vez, tratou você da seguinte forma: a) Forçou-a fisicamente a manter relações sexuais quando você não queria? b) Você teve relação sexual porque estava com medo do que ele(a) pudesse fazer? c) Forçou-a a uma prática sexual degradante ou humilhante?

A violência na gestação foi definida pela resposta sim à pergunta: Algumas destas vezes (que viveu alguma das situações descritas acima) você estava grávida? Portanto, o evento denominado por violência na gestação, refere-se, mais precisamente, aos casos em que a violência ocorreu também na gestação, mas não exclusivamente neste período.

A repetitividade dos episódios ou a recorrência da violência foi analisada a partir do número de vezes que ocorreu o episódio classificado em três categorias: uma, poucas e muitas vezes.

Baseando-se em uma análise preliminar da violência psicológica quanto à sua forma exclusiva ou combinada com a violência física ${ }^{23}$, criou-se um gradiente de gravidade, considerando-se moderada aquela que inclui exclusivamente os atos de insultos e humilhações (itens $a$. e $b$ da variável, como acima) e grave aquela que inclui ao menos um dos demais atos (itens $c$ [intimidação] e $d$ [ameaças]). No caso da violência física, considera-se moderada aquela caracterizada por tapas, empurrões, tranco ou chacoalhões (itens $a$ e $b$ da variável, como acima) e grave aquela caracterizada pelos demais itens (socos, chutes, surras, estrangulamento ou queimaduras, ameaça ou uso de arma de fogo, faca ou qualquer outro tipo de arma) ${ }^{22}$.

Para confecção e limpeza do banco de dados foi utilizado o programa FOXPRO 2.6 para Windows. A entrada de dados deu-se por meio de uma dupla digitação, seguida por checagem de consistência dos dados.
O tratamento estatístico foi realizado usando os programas STATAä 8.0.

A análise descritiva para caracterização da amostra de usuárias foi realizada através do cálculo de medidas de tendência central (média aritmética) e de dispersão (desvio padrão) para as variáveis contínuas, e de freqüência para as variáveis categóricas. Calculou-se a estimativa da prevalência de violência na gestação com cálculo do intervalo de $95 \%$ de confiança $\left(\mathrm{IC}_{95 \%}\right)$. Para a estimativa das associações de variáveis explanatórias com a presença de violência na gestação foram calculadas as razões de prevalência (RP), com os respectivos $\mathrm{IC}_{95 \%}$, e realizados testes de quiquadrado (X2) ou qui-quadrado de tendência linear (no caso de variáveis ordenadas) para testar a significância estatística das associações. Admitiu-se nível de significância estatística $\mathrm{p}<0,05$.

Além da Razão de Prevalência também foi calculado o Odds Ratio (OR) bruto das variáveis independentes estudadas. Em seguida foi feita análise multivariada através da construção de um modelo de regressão logística, com cálculo do OR ajustado. Foram incluídas no modelo as variáveis que apresentaram associação com a ocorrência de VPI na gestação com $\mathrm{p}<0,20$, bem como aquelas com relevância teórica para a análise. Permaneceram no modelo as variáveis independentes que se mantiveram associadas ao desfecho após o ajuste por todas as variáveis incluídas $(\mathrm{p}<0,05$ no teste de Wald) e aquelas consideradas relevantes pela literatura. A contribuição de cada variável para o modelo foi testada com o uso do teste da razão de verossimilhança (likelihood ratio test) e o ajuste do modelo foi testado com o uso do teste de ajuste de Hosmer-Lemeshow.

\section{Resultados}

\section{Descrição da amostra}

Das 1922 mulheres entrevistadas, notase que $39,5 \%$ tinham entre 5 e 8 anos de estudo, $42,5 \%$ pertenciam ao estrato 
socioeconômico D, 76,8\% eram casadas ou amigadas, $62,2 \%$ eram católicas e $51,5 \%$ se declaram de cor de pele branca. A média de idade foi de 32,1 anos, com desvio padrão (DP) de 9,0 anos. No que se refere às características de saúde reprodutiva e sexual, 50,5\% das usuárias iniciaram sua vida sexual entre 15 e 18 anos (média $=18,1$ anos e o DP = 6,0 anos) e 45,3\% tiveram de duas a três gestações. A média de filhos nascidos vivos foi de 2,1 filhos, com DP de 1,7 filhos. Das 1922 mulheres, 429 (22,3\%) tiveram um aborto, $119(6,2 \%)$ tiveram dois abortos, $50(2,6 \%)$ tiveram três ou mais abortos, $14,9 \%$ tiveram filhos com peso abaixo de $2,5 \mathrm{~kg}$ e $3,6 \%$ tiveram um ou mais filhos nascidos mortos.

Quanto à negociação de uso de camisinha, 22,5\% das usuárias revelaram que seu parceiro mais recente ou atual já se recusou a usar camisinha para prevenir alguma doença. Em relação às condições de saúde mental, 46,6\% das usuárias apresentam sintomatologia comum aos quadros de depressão, ansiedade ou transtornos mentais com queixas somáticas.

\section{Prevalência da violência por familiar e por parceiro na vida e na gestação}

A violência psicológica constitui a forma de violência familiar mais freqüente na população estudada (26,5\%), seguida pela física $(13,5 \%)$, sendo que, para muitas usuárias que sofrem estes tipos de violência, são eventos que ocorrem "muitas vezes na vida” (14,8\% sofrem VPI psicológica muitas vezes e $6,9 \%$ sofrem VPI física). A violência sexual por familiar é referida por $3,1 \%$ da amostra. A violência psicológica por familiar na gestação foi referida por $7,8 \%$ das usuárias e a física por 2,0\%.

Do total de mulheres entrevistadas $(n=1922), 60,6 \%$ (1.165) sofreram algum tipo de VPI na vida, sendo que $52,0 \%$ sofreram algum tipo de violência psicológica, 40,5\% violência física e $20,4 \%$ violência sexual. A violência psicológica conjugada com a física é o tipo de VPI mais freqüente entre as entrevistadas $(19,1 \%)$, seguido por violência só psicológica $(15,1 \%)$ e pela conjugação dos três tipos de violência; psicológica, física e sexual (14,4\%).

No que se refere à recorrência dos episódios de violência, para todos os tipos de violência, a categoria 'uma única vez na vida’ é a mais freqüente. Todavia, número expressivo das mulheres refere episódios repetitivos (muitas vezes) de violência psicológica $(14,1 \%)$, física $(5,1 \%)$ e sexual $(2,8 \%)$. Em termos da gravidade dos episódios da violência psicológica e física, observa-se que 36,2\% sofrem VPI psicológica grave e $24,3 \%$ sofrem VPI física grave. Em relação à violência na gestação, 20,0\% das entrevistadas afirmaram ter sofrido qualquer tipo de violência na gestação $\left(I C_{95 \%} 18,2\right.$ a 21,8$)$.

\section{Variáveis associadas à VPI na gestação}

As Tabelas 1, 2 e 3 resumem as análises univariadas realizadas tomando a VPI na gestação como variável dependente (presença ou ausência). A Tabela 1 referese às análises univariadas entre a freqüência da violência física e psicológica por familiar na vida e na gestação e a variável dependente. A Tabela 2 refere-se às análises entre a variável de desfecho e as características sócio-demográficas e a Tabela 3 contém os resultados das análises univariadas entre a variável de desfecho e as variáveis independentes relativas às características de saúde reprodutiva, sexual e mental.

\section{Análise multivariada}

$\mathrm{Na}$ análise multivariada, foi contrastada a variável dependente "violência durante a gestação", dicotômica (presença ou ausência) contra 14 variáveis que, em análise univariada, estiveram associadas à VPI na gestação: recorrência da violência psicológica, física e sexual por familiares; número de gestações; número de filhos nascidos vivos; número de abortos; número de filhos com baixo peso ao nascer; idade da primeira relação sexual; recusa de uso 
Tabela 1 - Razão de prevalência de VPI na gestação e violência perpetrada por familiar entre as usuárias que já engravidaram ( $\mathrm{n}=1922)$.

Table 1 - Prevalence ratio for IPV in pregnancy and violence perpetrated by a relative among users that have already been pregnant $(n=1,922)$

\begin{tabular}{|c|c|c|c|c|c|c|}
\hline Variáveis & Total & $\begin{array}{c}\text { VPI na } \\
\text { gestação }\end{array}$ & $\%$ & $\mathrm{RP}$ & $\mathrm{IC}_{95 \%}$ & $P$ \\
\hline \multicolumn{6}{|c|}{ Recorrência da violência psicológica por familiar } & $0,000^{*}$ \\
\hline Não sofreu & 1.413 & 242 & 17,1 & 1 & & \\
\hline Uma vez & 129 & 31 & 24,0 & 1,40 & 1,01 a 1,95 & \\
\hline Poucas vezes & 95 & 24 & 25,3 & 1,47 & 1,02 a 2,12 & \\
\hline Muitas vezes & 285 & 87 & 30,5 & 1,78 & 1,44 a 2,20 & \\
\hline \multicolumn{6}{|c|}{ Recorrência da violência física por familiar } & $0,000^{*}$ \\
\hline Não sofreu & 1163 & 304 & 18,3 & 1 & & \\
\hline Uma vez & 78 & 22 & 28,2 & 1,54 & 1,06 a 2,23 & \\
\hline Poucas vezes & 49 & 9 & 18,4 & 1,00 & 0,55 a 1,83 & \\
\hline Muitas vezes & 132 & 49 & 37,1 & 2,03 & 1,59 a 2,59 & \\
\hline \multicolumn{6}{|c|}{ Violência psicológica por familiares na gestação } & $0,000^{*}$ \\
\hline Não sofreu violência psicológica & 1413 & 242 & 17,1 & 1 & & \\
\hline Não sofreu na gestação & 360 & 84 & 23,3 & 1,36 & 1,09 a 1,69 & \\
\hline Sofreu na gestação & 149 & 58 & 38,9 & 2,27 & 1,80 a 2,86 & \\
\hline \multicolumn{6}{|c|}{ Violência física por familiares na gestação } & 0,000 \\
\hline Não sofreu violência física & 1663 & 304 & 18,3 & 1 & & \\
\hline Não sofreu na gestação & 220 & 60 & 27,3 & 1,49 & 1,17 a 1,90 & \\
\hline Sofreu na gestação & 39 & 20 & 51,3 & 2,80 & 2,03 a 3,87 & \\
\hline
\end{tabular}

* p corresponde ao teste de Qui-quadrado de tendência linear [p corresponds to the linear trend chi-square test]

${ }^{* *}$ p corresponde ao teste de Qui-quadrado. [p corresponds to the chi-square test]

de preservativo pelo parceiro; transtorno mental comum; estado civil; escolaridade; raça/cor da pele e prática religiosa.

Dentre as variáveis testadas, foram mantidas no modelo aquelas que estiveram independentemente associadas à VPI na gestação (recorrência da violência psicológica por familiares; recorrência de violência física por familiares; número de gestações, idade da primeira relação sexual, recusa de uso de preservativo pelo parceiro, estado civil e transtorno mental comum) e uma variável que não esteve associada, mas que é tida como relevante pela literatura (escolaridade) (Tabela 4). Optou-se por manter esta última como variável de ajuste.

A variável prática religiosa não foi mantida no modelo, por agrupar na categoria outras práticas religiões bastante heterogêneas e pouco representativas na amostra (3,0\%), tais como o judaísmo, o islamismo, o candomblé e a umbanda. Esta categoria outras práticas apresentou associação com VPI na gestação, tornando difícil sua interpretação. Assim, aponta-se a importância de estudo que garanta a representatividade das diferentes práticas religiosas. Além disto, este estudo indica a relevância da produção de investigações mais detalhadas sobre a associação entre VPI na gestação e a prática evangélica pentecostal, por ela ter apresentado associação com o desfecho aqui estudado.

A variável relativa à raça/cor da pele também foi retirada do modelo, pois, embora a categoria cor amarela/mestiça esteja estatisticamente associada à VPI na gestação, ela representa pequeno percentual dos casos $(2,0 \%)$, provocando desajuste ao ser inserida no modelo (teste de Hosmer-Lemeshow). Assim, este estudo também indica a relevância de novas investigações sobre a VPI na gestação na população amarela/mestiça.

De acordo com este modelo, sofrer violência psicológica e física por familiar 
Tabela 2 - Razão de prevalência da VPI na gestação e características sócio-demográficas das usuárias que já engravidaram ( $\mathrm{n}=1922)$.

Table 2 - Prevalence ratio for IPV in pregnancy and socio-demographic features of users who have already been pregnant $(n=1922)$

\begin{tabular}{|c|c|c|c|c|c|c|}
\hline Variáveis & Total & $\begin{array}{c}\text { VPI na } \\
\text { gestação }\end{array}$ & $\%$ & $\mathrm{RP}$ & IC95\% & $P$ \\
\hline Estado civil & & & & & & $0,000^{* * *}$ \\
\hline Casada/amigada & 1.476 & 254 & 17,2 & 1 & & \\
\hline Namora/com relação & 184 & 47 & 25,5 & 1,48 & 1,13 a 1,95 & \\
\hline $\begin{array}{l}\text { Está sem parceiro, ou } \\
\text { namora sem relação }\end{array}$ & 262 & 83 & 31,7 & 1,84 & 1,49 a 2,27 & \\
\hline Estrato social* & & & & & & $\mathrm{P}<0,001^{* * *}$ \\
\hline B & 111 & 16 & 14,4 & 1 & & \\
\hline $\mathrm{C}$ & 644 & 103 & 15,9 & 1,11 & 0,68 a 1,80 & \\
\hline $\mathrm{D}$ & 816 & 175 & 21,4 & 1,48 & 0,93 a 2,38 & \\
\hline$E$ & 350 & 89 & 25,5 & 1,76 & 1,08 a 2,87 & \\
\hline Escolaridade & & & & & & $\mathrm{P}<0,001^{* * *}$ \\
\hline Nove ou mais anos & 470 & 66 & 14,0 & 1 & & \\
\hline 5 a 8 anos & 760 & 159 & 20,9 & 1,49 & 1,14 a 1,94 & \\
\hline 0 a 4 anos & 692 & 159 & 23,0 & 1,64 & 1,26 a 2,13 & \\
\hline \multicolumn{7}{|l|}{ Raça/ Cor da pele* } \\
\hline Branca & 990 & 181 & 18,3 & 1 & & \\
\hline Preta & 178 & 35 & 19,7 & 1,07 & 0,77 a 1,48 & \\
\hline Amarela,mestiça & 38 & 15 & 39,5 & 2,16 & 1,42 a 3,08 & $0,001^{* *}$ \\
\hline Parda/Mulata/morena & 715 & 153 & 21,4 & 1,17 & 0,96 a 1,42 & \\
\hline Prática religiosa & & & & & & $<0,001^{* * *}$ \\
\hline Católica & 1.196 & 205 & 17,1 & 1 & & \\
\hline Evangélica histórica & 58 & 10 & 17,2 & 1,00 & 0,56 a 1,79 & \\
\hline Evangélica pentecostal & 395 & 104 & 26,3 & 1,54 & 1,25 a 1,89 & \\
\hline Espírita Kardecista & 34 & 8 & 23,5 & 1,37 & 0,73 a 2,54 & \\
\hline Sem prática religiosa & 182 & 41 & 22,5 & 1,31 & 0,97 a 1,76 & \\
\hline Outras & 57 & 16 & 28,1 & 1,64 & 1,06 a 2,53 & \\
\hline Idade & & & & & & $0,547^{* * *}$ \\
\hline 40-49anos & 455 & 88 & 19,3 & 1 & & \\
\hline 30-39 anos & 582 & 119 & 20,4 & 1,06 & 0,83 a 1,35 & \\
\hline 20-29anos & 745 & 143 & 19,2 & 0,99 & 0,78 a 1,26 & \\
\hline 15-19 anos & 140 & 34 & 24,3 & 1,25 & 0,89 a 1,77 & \\
\hline
\end{tabular}

* válido para 1921 [valid for 1,921]

** p corresponde ao teste de Qui-quadrado [p corresponds to the chi-square test]

*** p corresponde ao teste de Qui-quadrado de tendência linear [p corresponds to the linear trend chi-square test]

muitas vezes na vida é fator associado à ocorrência de VPI na gestação (OR ajusta$\mathrm{da}=1,54$ e 1,98, respectivamente). Em relação às variáveis de saúde reprodutiva e sexual, ter mais de três gestações na vida, iniciar as relações sexuais antes dos 15 anos e ter recusa de preservativo pelo parceiro para prevenção de DSTs também foram características associadas à VPI na gestação $(\mathrm{OR}$ ajustada $=3,44 ; 1,98 ; 1,62$, respectivamente). Ter transtorno mental comum e estar sem parceiro ou com namorado sem relação sexual também são variáveis associadas à VPI na gestação (OR ajustada $=2,22 ; 2,16$, respectivamente).

\section{Discussão}

Este estudo, o primeiro a abordar a violência por parceiro íntimo na gestação com 
Tabela 3 - Razão de prevalência de VPI na gestação e fatores de saúde sexual, reprodutiva e mental de usuárias que já engravidaram ( $n=1922)$.

Table 3 - Prevalence ratio for IPV in pregnancy and sexual, reproductive and mental health factors of users who have already been pregnant $(n=1922)$

\begin{tabular}{|c|c|c|c|c|c|c|}
\hline Variáveis & Total & $\begin{array}{l}\text { Violência na } \\
\text { gestação }\end{array}$ & $\%$ & $\mathrm{RP}$ & IC95\% & $P$ \\
\hline Idade da primeira relação & & & & & & 0,000 \\
\hline 19 ou mais & 667 & 89 & 13,3 & 1 & & \\
\hline de 15 a 18 & 970 & 207 & 21 & 1,60 & 1,27 a 2,01 & \\
\hline Menos de 15 & 285 & 88 & 30,9 & 2,31 & 1,78 a 3,00 & \\
\hline Recusa condom & & & & & & 0,000 \\
\hline Nunca recusou & 1489 & 259 & 17,4 & 1 & & \\
\hline Já recusou & 433 & 125 & 28,9 & 1,66 & 1,38 a 2,00 & \\
\hline$\underline{N^{\circ} \text { de gestações }}$ & & & & & & 0,000 \\
\hline Uma & 537 & 55 & 10,2 & 1 & & \\
\hline Duas ou três & 870 & 174 & 20,0 & 1,95 & 1,47 a 2,59 & \\
\hline Quatro ou mais & 515 & 155 & 30,1 & 2,94 & 2,21 a 3,90 & \\
\hline № de filhos nascidos vivos & & & & & & 0,000 \\
\hline Nenhum & 229 & 28 & 12,2 & 1 & & \\
\hline Um ou dois & 1058 & 178 & 16,8 & 1,37 & 0,94 a 2,00 & \\
\hline Três ou quatro & 483 & 125 & 25,9 & 2,11 & 1,44 a 3,09 & \\
\hline Cinco ou mais & 152 & 53 & 34,9 & 2,85 & 1,89 a 4,29 & \\
\hline Baixo peso ao nascer & & & & & & $<0,001$ \\
\hline Nenhum filho & 1.635 & 306 & 18,7 & 1 & & \\
\hline Um ou mais & 287 & 78 & 27,2 & 1,45 & 1,71 a 1,80 & \\
\hline № de filhos nascidos mort & & & & & & 0,7574 \\
\hline Nenhum & 1.852 & 369 & 19,9 & 1 & & \\
\hline Um ou mais & 70 & 15 & 21,4 & 1,07 & 0,68 a 1,69 & \\
\hline № de abortos & & & & & & 0,000 \\
\hline Nenhum & 1324 & 230 & 17,4 & 1 & & \\
\hline Um & 429 & 100 & 23,3 & 1,34 & 1,08 a 1,65 & \\
\hline Dois & 119 & 31 & 26,0 & 1,49 & 1,08 a 2,07 & \\
\hline Três ou mais & 50 & 23 & 46,0 & 2,65 & 1,92 a 3,66 & \\
\hline Escore no SRQ* & & & & & & 0,000 \\
\hline Sem TMC & 1025 & 131 & 12,8 & 1 & & \\
\hline Com TMC & 896 & 253 & 28,2 & 2,20 & 1,82 a 2,67 & \\
\hline
\end{tabular}

*Válido para 1921 mulheres. [valid for 1,921 women]

usuárias de atenção primária no Brasil, estima que $20,0 \%$ das mulheres entrevistadas que já engravidaram sofreram algum episódio de VPI no período da gestação. Esta taxa é similar àquela obtida em estudo internacional com desenho semelhante ${ }^{14}$.

No entanto, esta prevalência está abaixo daquelas encontradas por outros autores. Castro e Ruíz ${ }^{24}$, por exemplo, constataram taxa de $32,1 \%$ para qualquer forma de violência na gestação entre usuárias gestantes, no Estado do México. A grande diferença da magnitude encontrada nesse estudo em comparação aos dados encontrados no presente trabalho pode ser atribuída tanto às diferenças amostrais quanto aos critérios de definição de casos. No estudo citado, além do uso de instrumentos diferentes para a identificação de casos (o Index of Spouse Abuse e o Severity of Violence Against Women), a amostra foi composta por mulheres no terceiro trimestre da gravidez. É possível que a experiência atual da gestação facilite a lembrança 
Tabela 4 - Odds ratio bruto e ajustado das características associadas à VPI na gestação $(\mathrm{n}=1921)$.

Table 4 - Gross and adjusted odds ratio of the features associated with IPV in pregnancy $(n=1,921)$.

\begin{tabular}{|c|c|c|c|c|c|}
\hline & $\mathrm{RP}$ & $\mathrm{OR}_{\text {bruto }}$ & $\mathrm{OR}_{\text {ajustado }}$ & $I C_{95 \%}$ & $\mathrm{P}^{*}$ \\
\hline Recorrência de violência psicológica por familiar na vida & & & & & 0,02 \\
\hline Não sofreu & 1 & 1 & 1 & & \\
\hline Uma vez & 1,40 & 1,53 & 1,56 & 0,98 a 2,46 & \\
\hline Poucas vezes & 1,47 & 1,64 & 1,54 & 0,92 a 2,60 & \\
\hline Muitas vezes & 1,78 & 2,13 & 1,54 & 1,10 a 2,14 & \\
\hline Recorrência de violência física por familiar na vida & & & & & 0,02 \\
\hline Não sofreu & 1 & 1 & 1 & & \\
\hline Uma vez & 1,54 & 1,76 & 1,28 & 0,73 a 2,25 & \\
\hline Poucas vezes & 1,00 & 1,01 & 0,87 & 0,40 a 1,92 & \\
\hline Muitas vezes & 2,03 & 2,64 & 1,98 & 1,30 a 3,03 & \\
\hline Número de gestações & & & & & $<0,001$ \\
\hline Uma & 1 & 1 & 1 & & \\
\hline Duas ou três & 1,95 & 2,19 & 2,43 & 1,73 a 3,43 & \\
\hline Quatro ou mais & 2,94 & 3,77 & 3,44 & 2,39 a 4,96 & \\
\hline Idade da primeira relação sexual & & & & & $<0,001$ \\
\hline 19 anos ou mais & 1 & 1 & 1 & & \\
\hline 15 a 18 anos & 1,60 & 1,76 & 1,51 & 1,13 a 2,01 & \\
\hline Antes dos 15 anos & 2,31 & 2,90 & 1,98 & 1,37 a 2,85 & \\
\hline Recusa de uso de condom pelo parceiro & & & & & $<0,001$ \\
\hline Nunca & 1 & 1 & 1 & & \\
\hline Já recusou & 1,66 & 1,93 & 1,62 & 1,24 a 2,11 & \\
\hline Estado civil & & & & & $<0,001$ \\
\hline Casada/amigada & 1 & 1 & 1 & & \\
\hline Namora com relação sexual & 1,48 & 1,65 & 1,99 & 1,35 a 2,93 & \\
\hline Sem parceiro/ namora sem relação & 1,84 & 2,23 & 2,22 & 1,62 a 3,04 & \\
\hline Transtorno mental comum (SRQ) & & & & & $<0,001$ \\
\hline Não & 1 & 1 & 1 & & \\
\hline Sim & 2,20 & 2,68 & 2,16 & 1,68 a 2,77 & \\
\hline Escolaridade & & & & & 0,20 \\
\hline Nove ou mais anos & 1 & 1 & 1 & & \\
\hline Cinco a oito anos & 1,49 & 1,62 & 1,33 & 0,95 a 1,87 & \\
\hline Zero a quatro anos & 1,64 & 1,83 & 1,32 & 0,93 a 1,88 & \\
\hline
\end{tabular}

* $p$ referente ao teste da razão de verossimilhança/p refers to likelihood ratio test)

Teste de ajuste do modelo (Hosmer-Lemeshow): $\mathrm{p}=0,31 /$ Hosmer-Lemeshow model adjustment tests: $p=0.31$

e a revelação das entrevistadas sobre a violência sofrida neste período.

Moraes e Reichenheim ${ }^{7}$ também obtiveram prevalência bastante alta de qualquer tipo de VPI na gestação entre usuárias de três maternidades públicas do Rio de Janeiro, estimada em $63,4 \%$, ainda que esta taxa deva-se, sobretudo, à grande magnitude de violência psicológica encontrada relativamente às demais. Estas diferenças em relação ao presente estudo podem ser atribuídas à amostra, às condições diversas da entrevista (puérperas em materni- dades até 24 hs após o parto), ou mesmo a diversidades socioculturais por se tratar de outra cidade brasileira. O estudo com puérperas permite melhor investigação do período gestacional como um todo, aumentando a chance de identificação de situações de violência e, sobretudo, daquelas formas de VPI de menor gravidade.

Assim, é possível que no presente estudo tenha-se estimado principalmente as violências de intensidade mais graves e na sua forma física, dada a distância no tempo entre a gestação e a data da entrevista. 
Nesse sentido, o presente achado encontra-se próximo do leque de variação das violências físicas dos estudos latino-americanos ${ }^{7,24}$. Este viés de memória, todavia, reforça a subnotificação de casos, já bem reconhecida pela literatura como questão importante em pesquisas sobre violência doméstica. Tal subnotificação também é verificada relativamente à condição de coleta de dados, à banalização do fenômeno entre as mulheres e à evocação sentimentos de vergonha, culpa ou embaraço ${ }^{25}$. Esses fatores foram, nesta pesquisa, alvo de especial atenção e tratamento, seguindo recomendação da literatura ${ }^{22,25}$, a fim de minimizar a subinformação. Assim, quer pelo viés de memória, quer pela subinformação, reconhece-se que, mesmo diante de alta magnitude das prevalências encontradas, ainda podemos ter uma subestimação do fenômeno.

O estudo revela que o fenômeno é parte de um padrão de violência mais amplo, que inclui sofrer violência repetidas vezes por familiares ao longo da vida e na gestação, coincidindo plenamente com os achados reportados em diversas fontes ${ }^{5,6,24}$.

As mulheres solteiras, aquelas com parceiros sem relacionamento sexual e aquelas com namorado com relação sexual, mas sem coabitação, apresentam maior prevalência de VPI na gestação do que aquelas que convivem com os parceiros, confirmando achados de outros estudos $^{14,26,27}$. Por se tratar do estado civil da mulher no momento da entrevista e não necessariamente daquele no momento dos atos violentos, pode-se supor que as mulheres que vivem situações de VPI na gestação tendem a não estar atualmente coabitando com seus parceiros violentos. Isto pode ocorrer porque deixaram de viver com eles ou porque já não viviam quando engravidaram.

Ter mais de três gestações também é um componente fortemente associado à violência neste período. A literatura confirma que a mulher em situação de violência tem maiores chances de ter quatro ou mais filhos do que as mulheres que não sofrem violência ${ }^{14,28}$. Embora alguns autores sugiram que se interprete esta associação no sentido de a violência ser decorrência do maior estresse vivido por famílias com muitos filhos, um estudo em particular sugere interpretação inversa ${ }^{28}$. Ele constata que cerca de $50 \%$ de toda violência física se inicia nos dois primeiros anos da relação conjugal e $80 \%$ dentro dos quatro primeiros anos, isto é, antes do alto número de filhos. Desta forma, entende-se que a violência precede o alto número de filhos, podendo ser considerada em si um fator de risco para se ter mais filhos: por exemplo, por dificultar a adoção de medidas contraceptivas.

Embora, em análise univariada, se tenha constatado associação entre o número elevado de abortos (3 ou mais) e a violência por parceiro íntimo na gestação, no modelo multivariado, quando se inclui a variável número de gestações, a associação do desfecho com a categoria 3 ou mais abortos perde sua significância estatística. Este resultado sugere que o número de abortos e o número de gestações são variáveis que medem eventos relacionados, gerando 'superajuste' quando colocadas no modelo. Além disto, apenas 50 mulheres referem 3 ou mais abortos, tornando muito instáveis as estimativas ligadas a este estrato.

Ter tido relação sexual antes dos 19 anos de idade também está associado à VPI na gestação, sobretudo quando ela ocorre antes dos 15 anos. É possível analisar este achado no sentido de que as mulheres que experimentam situações de violência doméstica na família de origem procurem mais cedo na vida um vínculo amoroso como meio de saída ou fuga do contexto familiar, do que aquelas que não sofrem violência neste contexto. É também possível que este dado indique que a primeira relação sexual seja ela mesma um ato de violência sexual na infância ou na adolescência perpetrada por um membro da família. Pode se referir também à auto-estima da mulher que sofre violência no núcleo familiar. A literatura indica que a ex- 
periência de violência neste contexto tem importante impacto na vida emocional da mulher e na sua auto-estima, provocando comportamentos de risco para sua saúde e, provavelmente, envolvimento com múltiplos parceiros em idade jovem.

As mulheres cujos parceiros já recusaram uso de preservativo apresentam maior prevalência de VPI na gestação. Este dado reforça a idéia de que aquelas que sofrem VPI têm menor poder de negociação e autonomia dentro da relação amorosa e estão mais suscetíveis às DSTs.

A associação entre TMC e VPI na gestação, embora indique presença de sintomatologia comum aos transtornos depressivos e de ansiedade, não nos permite inferências causais, tanto pela própria natureza do estudo de corte transversal, como pelo fato de o SRQ-20 avaliar a sintomatologia das últimas quatro semanas e a ocorrência de VPI na gestação poder ter ocorrido muitos anos antes.

No modelo de regressão logística, a escolaridade da mulher não se manteve associada ao desfecho, diferindo de achados produzidos em outras pesquisas ${ }^{12,14,24}$. Este dado sugere que as questões de saúde reprodutiva, sexual e mental têm maior força de associação sobre a VPI na gestação do que as questões socioeconômicas. Não se trata de desconsiderar o papel das precárias condições de vida, do menor acesso ao sistema educacional e da baixa renda na ocorrência da violência. Mas significa observar que, em relação à VPI na gestação, as questões de gênero relacionadas à autonomia de negociação de preservativos, ao número de gestações, à idade de início das atividades sexuais e ao estado civil parecem ter maior impacto.

É importante reforçar limitações metodológicas deste estudo. A partir de dados produzidos com o objetivo de estimar as prevalências das violências ocorridas na vida e no último ano, para as usuárias em geral dos serviços de saúde, além da presença de agravos à saúde associados e uso dos serviços de saúde, a abordagem da VPI durante a gestação constituiu propósito subjacente e não central de seu desenho. Portanto, os dados produzidos sobre esta questão, embora importantes para um primeiro panorama da prevalência em atenção primária, permitindo-se pensar melhor os serviços, não viabilizam análise mais detalhada sobre o tipo de violência (psicológica, física ou sexual), a gravidade e a recorrência da violência vivida especificamente durante a gestação. Eles fornecem informações exclusivamente sobre a presença ou ausência de algum episódio de violência durante este período.

Outra restrição desse estudo refere-se também à ausência de informações sobre o parceiro. Este fato limita a análise sobre algumas características do relacionamento que podem entrar em jogo nas situações de violência, sobretudo tendo em vista um dos resultados dessa pesquisa acerca da importância das questões de gênero enquanto parte das origens da violência na gestação. A ausência das informações sobre o parceiro dificulta a compreensão do modo como as trajetórias de vida e a inserção social dos indivíduos se intersectam, favorecendo a ocorrência de violência.

\section{Referências}

1. Campbell J, García-Moreno C, Sharps P. Abuse during pregnancy in industrialized and developing countries. Violence Against Women 2004; 7: 770-89.

2. Nasir, J; Hyder A Violence against pregnant women in developing countries - Review of evidence. Eur J Public Health 2003; 13(2): 105-07.
3. Gazmararian J, Lazorick S, Spitz Am, Ballard Tj, Saltzman Le, Marks Js. Prevalence of Domestic Violence Against Pregnant Women. JAMA 1996; 275: 1915-20.

4. Jasinski, J.L. Pregnancy and domestic violence: A review of the literature. Trauma, Violence \& Abuse, 2004; 5(1): 47-64. 
5. Heise L, Ellsberg M, Gottemoeller M. Ending Violence Against Women. Population Reports, Series L, N 11. Baltimore, Johns Hopkins University School of Public Health, Population Information Program. Dezembro, 1999.

6. Menezes TC, Amorim MMR, Santos C, Faúndes A. Violência física doméstica e gestação: resultados de um inquérito no puerpério. RBGO 2003; 25(5): 309-16.

7. Moraes CL, Reichenheim ME. Domestic violence during pregnancy in Rio de Janeiro. Int J Gynaecol Obstet 2002; 79: 269-77.

8. Coid J, Petruckevitch A, Chung W, Richardson J, Moorey S, Feder G. Abusive experiences and psychiatric morbidity in women primary care attenders. $\mathrm{BrJ}$ Psychiatry 2003; 183: 332-9.

9. Lovisi GM, Lopez JR, Coutinho ES, Patel V. Poverty, violence and depression during pregnancy : a survey of mothers attending a public hospital in Brazil. Psychol Med 2005; 35(10): 1485-92.

10. Mcfarlane J, Parker B, Soeken K. Physical abuse, smoking, and substance use during pregnancy: Prevalence, interrelationships, and effects on birth weight. JObstet Gynecol Neonatal Nurs 1996; 25(4):313-20.

11. Murphy CC, Schei B, Myhr TL, Du Mont J. Abuse: risk factor for low birth weight? A systematic review and meta-analysis. Can Med Assoc J2001; 164(11): 1567-72.

12. Mcfarlane J, Parker B, Soeken K, Bullock L. Assessing for abuse during pregnancy: Frequency and extent of injuries and entry into prenatal care. JAm Med Assoc 1992; 267: 3176-8.

13. Curry Ma, Perrin N, Wall E. Effects of abuse on maternal complications and birth weight in adult adolescent women. Obstet Gynecol 1998; 92(4): 530-4.

14. Coker Al, Sanderson M, Dong B. Partner Violence during pregnancy and risk of adverse pregnancy outcomes. Paediatr Perinat Epidemiol 2004; 18(4): 260-9..

15. Mcfarlane J. Campbell Jc, Sharps P. Watson K. Abuse During Pregnancy and Femicide: Urgent Implications for women's health. Am Coll Obstet Gynecol 2002; 100(1): 2736.

16. Durand, J.G. Gestação e Violência: Estudos com usuárias de serviços públicos de saúde da Grande São Paulo [dissertação de mestrado]. São Paulo: Departamento de Medicina Preventiva, Faculdade de Medicina da USP; 2006.

17. Schraiber L B, D’Oliveira A F, Couto M T, Pinho A, Hanada H, Felicíssimo A, et al.. Ocorrência de casos de violência doméstica e sexual nos serviços de saúde em São Paulo e desenvolvimento de tecnologia de atendimento para o programa de saúde da mulher. São Paulo: FMUSP; 2003. (Relatório final de pesquisa a FAPESP).
18. Schraiber L B, D'Oliveira A F, Couto M T, Hanada H, Kiss L B, Durand JG, et al. Violência contra mulheres entre usuários de serviços públicos da Grande São Paulo. Rev Saúde Pública 2007; 41(3): 359-67.

19. Mcauley J, Kern DE, Kolodner K, Dill L, Schoeder AF, Ryden J, et al. The "Battering Syndrome": prevalence and clinical characteristics of Domestic Violence in Primary Care Internal Medicine Practices. Ann Intern Med 1995; 123: 737-46.

20. ABIPEME (Associação Brasileira de Institutos de Pesquisa de Mercado). Critérios de classificação Econômica. Junho de 2001. Disponível em http:/ /www.abipeme.org.br. Acessado em junho de 2001.

21. Mari J; Williams P. A validity study of a psychiatric screening questionnaire (GHQ-12 and SRQ-20) in Brazil using Relative Operating Characteristic (ROC) analysis. Psychol Med 1986;15: 651-9.

22. Garcia-Moreno C, Jansen HA, Ellsberg M, Heise L, Watts $\mathrm{CH}$, on behalf of the WHO Multi-country Study on Women's Health and Domestic Violence against Women Study Team. The Lancet 2006; 368(9543): 1260-9.

23. Schraiber LB; D’oliveira AF ; França Jr I; Diniz CSG; Portella AP; Ludermir AB et al. WHO Multi Country Study on Women's Health and Domestic Violence against Women. Final Report. BRAZIL. São Paulo: FMUSP, 2002. (Relatório final de pesquisa da Organização Mundial da Saúde -WHO)

24. Castro, R. E Ruíz, A. Prevalencia y severidad de la violencia contra mujeres embarazadas. Rev Saúde Pública 2004; 38(1): 62-70.

25. Ellsberg MC, Heise L, Peña R, Agurto S, Winkvist A. Researching Domestic Violence Against Women: Methodological and Ethical Considerations. Stud Fam Plann 2001; 32(1): 3-15.

26. Bacchus L, Mezey G, Bewley S. Domestic Violence: prevalence in pregnant women and associations with physical and psychological health. Eur J Obstet Gynecol Reprod Biol 2003; 113: 6-11.

27. Johnson, J.K., Haider, F., Ellis, K., Hay, D.M., Lindow, S.W. The prevalence of domestic violence in pregnant women. Int J Obstet Gynecol 2003; 110: 272-5.

28. Ellsberg, Mc, Penã R, Herrara A, Liljestrand J, Winkvist A. Wife Abuse Among Women of Childbearing Age in Nicaragua. Am J Public Health 1999; 89: 241-4.

Recebido em: 22/06/07 Versão final reapresentada em: 03/08/07 Aprovado em: 13/08/07 